\title{
Os impactos no pré-natal e na saúde mental de gestantes durante a pandemia de COVID-19: uma revisão narrativa
}

\author{
Impacts on prenatal care and mental health of pregnant women during the COVID-19 \\ pandemic: a narrative review
}

Impactos en la atención prenatal y la salud mental de las mujeres embarazadas durante la pandemia COVID-19: una revisión narrativa

Ana Luiza Miranda da Silva ${ }^{1 *}$, Angélica Silvério Oliveira², Brena Juliana Silva Ruas ${ }^{3}$, Lara Pinheiro Leão Piuzana Barbosa ${ }^{4}$, Maria Eduarda de Paula Adário Landim ${ }^{5}$, Rogério Reis Bruno ${ }^{5}$, Samira dos Santos Ferreira de Freitas ${ }^{6}$, Talita Miranda Santos ${ }^{7}$, Tiago Picolo Fernandes ${ }^{8}$, Tatiane Carolina Batista Nacif Roza9.

\section{RESUMO}

Objetivo: Apresentar as implicações que a pandemia da COVID-19 trouxe para a adesão à assistência prénatal e para a saúde mental de gestantes e puérperas. Revisão bibliográfica: A necessidade da adoção de medidas para evitar a disseminação do vírus, o receio da contaminação e suscetibilidade para desfecho em parto prematuro, ruptura prévia de membranas, restrição do crescimento intrauterino e natimortalidade, resultou em uma queda significativa na adesão à assistência pré-natal. Essa conjuntura ocorre devido ao cancelamento de consultas, a dificuldade de acesso a telemedicina e a necessidade da prorrogação em até 14 dias em casos de confirmação ou suspeita de infecção, o que pode comprometer a segurança e o bemestar materno-fetal. Associado a isso, observa-se a intensificação de sentimentos como medo e solidão, com recrudescimento de sintomas depressivos e ansiosos. Considerações finais: Diante disso, é de vital importância enfatizar a importância da assistência pré-natal, da flexibilização do acesso a essas consultas e do estabelecimento de um acompanhamento médico e familiar humanizado, a fim de garantir um suporte emocional ainda mais necessário no contexto hodierno.

Palavras-chave: COVID-19, Cuidado pré-natal, Saúde mental.

\begin{abstract}
Objective: To present the implications that the COVID-19 pandemic brought to prenatal care adherence and to the mental health of pregnant and postpartum women. Bibliographic review: The need to adopt measures to prevent the spread of the virus, the fear of contamination and susceptibility to the outcome with premature birth, previous rupture of membranes, intrauterine growth restriction and stillbirth, resulted in significant decline to prenatal care adherence. This conjuncture is due to the cancellation of appointments, the difficulty of accessing telemedicine and the need for an extension of up to 14 days in the cases of confirmation or suspicion infection, which can compromise the safety and welfare of mothers and babies. Associated with that, there is an intensification of feelings such as fear and loneliness, with resurgence of depressive and anxiety symptoms. Final considerations: That said, it is very important to emphasize the necessity of prenatal care, flexible access to these consultations and the establishment of humanized medical and family follow-up, in order to garantee an emotional suport, even more important in today's context.
\end{abstract}

Key words: COVID-19, Prenatal care, Mental health.

\footnotetext{
${ }^{1}$ Faculdade de Minas (FAMINAS-BH), Belo Horizonte - MG. *E-mail: analuizam49@yahoo.com

2 Universidade do Estado de Mato Grosso (UNEMAT), Cáceres - MT.

${ }^{3}$ Universidade Estadual de Montes Claros (UNIMONTES), Montes Claros - MG.

${ }^{4}$ Universidade Federal de Viçosa (UFV), Viçosa - MG.

${ }^{5}$ Centro Universitário de Volta Redonda (UniFOA), Volta Redonda - RJ.

${ }^{6}$ Universidade Anhembi Morumbi (UAM), São Paulo - SP.

7 Universidade de Itaúna (UIT), Itaúna - MG.

8 Universidade Nove de Julho (UNINOVE), Bauru - SP.

${ }^{9}$ Centro Universitário de Caratinga UNEC (UNEC), Caratinga - MG.
} 


\section{RESUMEN}

Objetivo: Presentar las implicaciones que la pandemia COVID-19 trajo a la adherencia a la atención prenatal y a salud mental de las mujeres embarazadas y en posparto. Revisión bibliográfica: La necesidad de adoptar medidas para prevenir la propagación del virus, el temor a la contaminación y la susceptibilidad al resultado en el parto prematuro, la ruptura previa de membranas, la restricción del crecimiento intrauterino y la muerte fetal, resultó en una caída significativa en la adherencia a la atención prenatal. Esta situación se debe a la anulación de citas, la dificultad para acceder a la telemedicina y la necesidad de una prórroga de hasta 14 días en los casos de confirmación o sospecha de infección, que pueden comprometer la seguridad y el bienestar de madres y bebés. Asociado a esto, hay una intensificación de sentimientos como el miedo y la soledad, con un resurgimiento de síntomas depresivos y de ansiedad. Consideraciones finales: Dicho eso, es de vital importancia enfatizar la necesidad de la atención prenatal, el acceso flexible a estas consultas y el establecimiento de un seguimiento médico y familiar humanizado, a fin de garantizar un apoyo emocional aún más necesario en el contexto actual.

Palabras clave: COVID-19, Atención prenatal, Salud mental.

\section{INTRODUÇÃO}

O surgimento da Coronavirus disease (COVID-19), infecção respiratória causada por uma nova cepa denominada coronavírus da Síndrome Respiratória Aguda Grave 2 (SARS-CoV-2), e sua rápida disseminação mundial, implicou na declaração de estado de pandemia em março de 2020 pelas autoridades da Organização Mundial de Saúde (OMS). A partir de então, foram necessárias a adoção de medidas de proteção contra a doença, principalmente $o$ isolamento social. Essas medidas impactaram especialmente a rotina de gestantes e puérperas, pois, além das alterações hormonais e novos desafios inerentes à maternidade, também culminou em mudanças no atendimento pré-natal, no cuidado e aleitamento do Recém-Nascido (RN) (PAZ MS, et al., 2021).

Sabe-se que a gravidez por si só leva a maior suscetibilidade para doenças respiratórias devido ao aumento da demanda de oxigênio, elevação diafragmática e diminuição da complacência torácica o que, consequentemente, resulta em menor tolerância à hipoxemia. Tais alterações podem acarretar em parto prematuro, crescimento intrauterino restrito, ruptura prematura de membranas e natimortalidade. Por esses motivos, as gestantes foram incluídas nos grupos de risco para a COVID-19, sendo o Brasil um dos primeiros países a tomar essa decisão (CASTRO P, et al., 2020).

A assistência pré-natal é de suma importância à saúde das mulheres durante a gestação e o puerpério e está associada a melhores desfechos perinatais. Porém, apesar de necessitarem de mais cuidados, as gestantes têm enfrentado dificuldades para realizar o acompanhamento pré-natal devido a cancelamento de consultas, consultas via teleatendimento ou adiamento por até nos casos de suspeita ou confirmação de infecção pelo COVID-19 (DING W, et al., 2021).

Estudo de Paixão JN, et al. (2021) demonstrou que, além desses obstáculos, outros fatores como a necessidade de isolamento trouxeram ainda mais dificuldades às gestantes, dificultando o contato com sua rede de apoio (familiares e amigos), além da necessidade de lidar com uma sobrecarga de notícias e informações a respeito do crescente número de casos confirmados e óbitos causados pelo coronavírus. Logo, todos esses fatores têm contribuído para o aumento significativo de sintomas depressivos e ansiosos em gestantes, em relação aos números pré-pandêmicos nesse mesmo grupo.

Outros estressores impostos pela pandemia que contribuem para diminuição da qualidade de vida das gestantes incluem dificuldades financeiras, maior risco de violência doméstica e atividades remotas de trabalho e escola (GONZALEZ RC e ALDERCICE F, 2020).

No caso de pacientes confirmadas para COVID-19, também fica prejudicado o desenvolvimento do vínculo materno-fetal, devido às dificuldades no aleitamento materno e privação do contato pele a pele com o RN pelo risco de contaminação viral do bebê. Todas essas condições que geram ansiedade podem acarretar em complicações materno-fetais importantes como parto prematuro, restrição de crescimento intrauterino, 
complicações obstétricas durante o parto e alterações no desenvolvimento neuropsicomotor da criança, como Transtorno do Déficit de Atenção e Hiperatividade (TDAH) (TRAPANI JÚNIOR A, et al., 2020; DING W, et al., 2021).

Diante desse contexto, o presente estudo teve como objetivo refletir acerca das modificações impostas ao atendimento gestacional durante a pandemia, bem como os impactos na saúde mental e física das gestantes provocados por esse contexto.

\section{REVISÃO BIBLIOGRÁFICA}

O pré-natal tem por objetivo garantir o bem-estar e a segurança materno-fetal, por meio de consultas periódicas, avaliação física e de exames complementares, a partir de uma escuta qualificada, a fim de diagnosticar ou amenizar precocemente riscos à saúde da gestante e do bebê (FONTANA AP, et al., 2017). Segundo recomendações do Ministério da Saúde (2013), a assistência pré-natal deve ser fundamentada em condutas acolhedoras, propiciando o vínculo e o fácil acesso a serviços de saúde de qualidade, bem como estimula-se o desenvolvimento de ações educativas e preventivas para detecção precoce de patologias e de situações de risco gestacional.

Durante a gestação, o organismo da mulher passa por diversas alterações fisiológicas. Entre elas, são observadas modificações da função cardiopulmonar, aumento do diafragma, aumento do consumo de oxigênio e edema da mucosa das vias respiratórias, que resultam em intolerância à hipóxia e maior susceptibilidade a complicações respiratórias e sistêmicas virais. Esse fato já foi observado em epidemias como o Severe Acute Respiratory Syndrome Coronavirus (SARS-CoV), Middle East Respiratory Syndrome Coronavirus (MERS-CoV) e na influenza pelo H1N1, em que houve um elevado número de partos pré-termo, além de restrição de crescimento e morte fetal (RAMALHO C, et al., 2021; CASTRO P, et al., 2020).

Com a atual pandemia de COVID-19 e a necessidade de diminuir as graves consequências da doença e o extenso número de óbitos, foram identificados grupos de risco para complicações e morte. Dentre estes estão os idosos, pneumopatas, portadores de doenças crônicas, profissionais da área de saúde e, mais recentemente, as gestantes. A inclusão tardia das gestantes como grupo de risco, provavelmente se deu em virtude da baixa frequência de gestantes na China, primeiro país a ser acometido pela doença (SOUZA AR e AMORIM MR, 2021).

O comportamento da COVID-19 difere em cada região do mundo, devido influências socioeconômicas e geográficas (MINISTÉRIO DA SAÚDE, 2020). No início da pandemia, a maioria dos relatos na literatura mostravam que grande parte das gestantes apresentavam quadros clínicos leves ou moderados e que uma baixa porcentagem destas necessitavam de suporte ventilatório ou cuidados em unidade de terapia intensiva (UTI). Porém, com o aumento do número de casos mundialmente foi verificado maior risco de complicações maternas, principalmente no último trimestre da gravidez e no puerpério (RASMUSSEN SA e JAMIESON DJ, 2020). No Brasil, desde abril de 2020, estudos vêm sendo publicados alertando para o risco de morte materna pelo vírus (NAKAMURA-PEREIRA M, et al., 2020; TAKEMOTO MS, et al., 2020; SOUZA AR e AMORIM MR, 2021).

Pesquisas têm evidenciado que grávidas, quando infectadas pelo novo coronavírus, têm cerca de doze vezes mais chances de hospitalização e duas vezes mais chances para necessidade de ventilação moderada (QEADAN F, et al., 2021; SANTOS DS, et al., 2020). Dados recentes do Observatório Obstétrico Brasileiro Covid-19 (OOBr Covid-19) mostraram que o número de mortes de grávidas e puérperas por COVID-19 ficou muito acima do registrado na população em geral, reforçando a necessidade da intensificação do cuidado a essas mulheres (FIOCRUZ, 2021).

Entretanto, nesse período de pandemia, diversas medidas foram impostas para o controle da doença alterando significativamente o modo de viver dos indivíduos e, por consequência, impactaram a assistência à saúde em todos os níveis de atenção (MALTA DC, et al., 2020). Visto que a principal forma de transmissão da COVID-19 ocorre através de gotículas respiratórias emitidas por pacientes infectados e por contato direto com os mesmos, o uso de máscaras, isolamento social, afastamento das atividades laborais e escolares, 
foram algumas das medidas de contenção viral adotadas (SILVA HN, et al., 2020; CASAGRANDE M, et al., 2020).

Uma importante questão constatada foi o abandono aos tratamentos e acompanhamentos médicos outrora instituídos, sendo observada uma queda de quase $46 \%$ no número de procedimentos clínicos, $44 \%$ na taxa de procedimentos pré-natais e queda de $36 \%$ no número de consultas médicas, quando comparado ao período anterior à pandemia (CHISINI LA, et al., 2021). Assim, toda assistência à saúde à mulher foi afetada pela pandemia, tanto pela priorização da assistência ao tratamento da COVID-19, quanto pelo receio de em procurar o serviço de saúde devido às incertezas e ao medo de sair de casa, aumentando a frequência de sinais e sintomas de ansiedade e depressão (SOUZA AR, et al., 2020).

De acordo com a Associação Paulista de Medicina, 64\% dos profissionais da área de ginecologia e obstetrícia afirmam que a rotina da assistência pré-natal foi alterada durante a pandemia. Segundo eles, $8 \%$ das mulheres não realizaram os exames subsidiários e $46 \%$ delas tiveram dificuldades para realizá-los. Neste contexto, além da gravidez já ser naturalmente um período de grande estresse e conflitos emocionais devido às mudanças hormonais sofridas, esses sentimentos são agravados pelas incertezas e o medo que cercam a infecção pela COVID-19, sendo essa a maior preocupação expressada pelas grávidas nas consultas (FEBRASGO, 2020).

O Brasil foi um dos primeiros países a incluir as gestantes e mulheres no período puerperal, que sofreram perdas fetais ou abortamento, como pertencentes ao grupo de risco para a COVID-19. Outra medida adotada pelo Ministério da Saúde foi a criação do "Manual de Recomendações para a Assistência À Gestante e Puérpera frente à Pandemia de Covid-19", de forma a orientar o acesso à saúde no decorrer da pandemia por meio de diretrizes, reduzindo as chances de contágio, morbimortalidade materna e agravos ao concepto (CASTRO P, et al., 2020; MINISTÉRIO DA SAÚDE, 2020).

De acordo com o Ministério da Saúde (2020), deve-se assegurar o pré-natal de todas as gestantes e, para otimização deste atendimento e diminuição da exposição ao risco, recomenda-se incluir as coletas de exames e ultrassonografias no dia da consulta presencial. Além disso, o intervalo de tempo entre as consultas deve ser determinando a partir da idade gestacional, a presença ou não de doenças maternas ou fetais, comorbidades e a evolução da gestação. A utilização da teleconsulta também pode ser uma opção de forma espaçar as consultas mantendo o cuidado, desde que garantido o adequado registro no prontuário da gestante.

A diretriz orienta, ainda, que as gestantes sem síndrome gripal e sem comorbidades, e que não fazem parte do grupo de risco, devem manter seis consultas de pré-natal, sendo uma no $1^{\circ}$ trimestre, duas no $2^{\circ}$ trimestre e três no $3^{\circ}$ trimestre. Já para as mulheres com sintomas gripais, recomenda-se que as consultas e exames de rotina sejam prorrogados em 14 dias e, se houver necessidade, que estas sejam atendidas de forma isolada das demais pacientes. Além disso, deve ser feito o registro sobre a suspeita de COVID-19 no pré-natal, para que a gestante possa ser monitorada pela equipe da atenção primária (MINISTÉRIO DA SAÚDE, 2020).

Em relação ao momento e à via de parto, não há nenhuma alteração nas recomendações para as mulheres com suspeita ou confirmação de COVID-19 no início da gravidez e que se recuperaram. Já para mulheres com suspeita ou confirmação da doença no terceiro trimestre que ainda não se recuperaram, recomenda-se adiar o parto até que um resultado negativo seja obtido na tentativa de evitar a transmissão para o neonato, se condições maternas e fetais permitirem. Além disso, a infecção por COVID-19 não é uma indicação para o parto, entretanto, em gestantes que evoluem com sintomas graves ou críticos, indica-se antecipação do parto e a realização de cesárea. Em gestantes com boas condições clínicas, sintomas leves e boa vitalidade fetal, o parto vaginal é seguro e recomendado (FEBRASGO, 2020).

Ainda no que diz respeito ao pré-natal, além da importância para a saúde materna e fetal, durante todo o acompanhamento gestacional é reforçado o vínculo da grávida com o profissional de saúde, trazendo assim, vantagens emocionais, uma vez que a futura mãe se sente mais acolhida pela equipe e mais confiante sobre a gestação (FONTANA AP, et al., 2017). As expectativas criadas nesse período, as mudanças emocionais, a 
ansiedade e o medo, associados ao processo de transformação e reestruturação da mulher em relação a maternidade, faz com que a grávida questione e reveja os conceitos a respeito de proteção e cuidado, sob a ótica do afeto, outro ponto que enfatiza a importância do acompanhamento à gestante, neste momento de tantas mudanças (PAIXÃO JN, et al., 2021).

A vivência da maternidade pode ser considerada como uma experiência solitária, uma vez que a mulher é encarregada pela sociedade como a principal, se não a única, responsável pelo cuidado e desenvolvimento saudável da criança (SILVA J e MELO MQ, et al., 2020). O isolamento social, como forma de prevenção da COVID-19, acaba por agravar este sentimento de solidão, uma vez que retira a mulher da sua rede de apoio, que ajudava nas tarefas domésticas, orientação e suporte emocional (ALMEIDA M, et al., 2021).

Mulheres grávidas no primeiro e terceiro trimestres da gravidez são extremamente vulneráveis, pois ocorrem mudanças hormonais, físicas, psicológicas e sociais. Com o contexto imposto pelo novo coronavírus, independente do contexto social, a construção da maternidade passada desde as escolhas de enxovais, berço, experiências de saberes compartilhadas com familiares e amigos foram suspensas. Fatos que acarretam ainda mais sintomas de ansiedade, com resultado para desafios psicológicos para mulheres grávidas e puérperas (PAIXÃO JN, et al., 2021; HESSAMI K, et al., 2020).

A ansiedade e a depressão acometem uma a cada sete mulheres no perinatal, entretanto, durante a gravidez e o período perinatal, $50 \%$ das mulheres com depressão ficam sem diagnóstico (HESSAMI K, et al., 2020). Chivers BR, et al. (2020) mostraram que as mulheres grávidas avaliadas durante a pandemia COVID19 relataram mais angústia e sintomas psiquiátricos do que as mulheres avaliadas antes da pandemia, principalmente na forma de sintomas de depressão e ansiedade. Os autores concluíram, ainda, que a falta de informações detalhadas e fidedignas pode exacerbar o risco de sofrimento psicológico e psicossocial nessas mulheres.

A redução das relações interpessoais entre parturiente e seus familiares pela restrição do número de acompanhantes no pré e pós-parto devido a pandemia são fatores que contribuem para o sentimento de solidão e aprofundamento da ansiedade e depressão, sentimentos recorrentes na gravidez, durante o período gravídico. Levando-se em consideração a importância de acompanhantes em todas as etapas do parto, como frisado na Lei do Acompanhante (Lei 11.108/2005), isolar a grávida, como vem acontecendo em alguns hospitais, além de ferir os seus direitos, prejudica também sua saúde mental (ESTRELA FM, et al., 2020).

Dessa forma, faz-se necessário a implantação de estratégias que visem a redução e os riscos do contágio, preservando o direito do acompanhante, mesmo que a gestante esteja com COVID-19. Dentre essas estratégias podem ser citadas a não permissão para revezamentos, a escolha de acompanhantes que não pertencem a grupos de risco para COVID-19, o estímulo de ligações de vídeo com os familiares e entrega de lembranças para que reduza um pouco do sentimento de solidão, realização de teste de triagem para síndromes gripais em pessoas que irão acompanhar a gestante (SOUZA RK, et al., 2016; MINISTÉRIO DA SAÚDE, 2020; PAIXÃO JN, et al., 2021)

Diante desse contexto, é de extrema importância a preocupação com os cuidados às gestantes e puérperas, visto que muitas que já apresentavam dificuldades de acesso ao pré-natal de qualidade, viu esse cenário piorar nesse período de pandemia. Assim, deve ser reforçada a importância do acompanhamento do pré-natal de forma a garantir a saúde da gestante e do feto durante a gravidez, mesmo que seja necessária uma reorganização para que as mulheres tenham um acompanhamento médico correto e um suporte emocional familiar adequado (SOUZA AR, et al., 2020; MINISTÉRIO DA SAÚDE, 2020). É importante, ainda, que $a$ atenção humanizada seja prioridade durante a gestação, mesmo com todos os problemas enfrentados durante a pandemia de COVID-19, de forma que seja possível que a mulher tenha uma vivência plena da maternidade, com segurança e cuidado (MINISTÉRIO DA SAÚDE, 2020).

\section{CONSIDERAÇÕES FINAIS}

O presente trabalho evidenciou como o cenário pandêmico provocado pela COVID-19 impactou o período gestacional de mulheres em todo o mundo, onde sentimentos de medo e ansiedade, inerentes à gestação, 
tornaram-se ainda mais intensos quando somados aos riscos impostos pelo vírus. A maior suscetibilidade a doenças respiratórias, o risco de parto prematuro, a restrição do crescimento intrauterino e a ruptura prematura de membranas, são exemplos de agravos que puderam ser confirmados como responsáveis por uma redução significativa na adesão à assistência pré-natal. Assim, reforça-se a importância da assistência no período gestacional para a saúde e o bem-estar materno-fetal, fazendo valer de recursos que facilitem o acesso das gestantes às consultas, levando em conta as dificuldades impostas em toda a rede assistencial atualmente.

\section{REFERÊNCIAS}

1. ALMEIDA M, et al. Pregnant women and COVID-19: isolation as a physical and psychic impact factor. Revista Brasileira de Saúde Materno Infantil, 2020; 20(2): 603-606.

2. BARROS M, et al. Relato de tristeza/depressão, nervosismo/ansiedade e problemas de sono na população adulta brasileira durante a pandemia de COVID-19. Revista Epidemiologia e Serviços de Saúde, 2020; 29(4): e2020427.

3. MINISTÉRIO DA SAÚDE. Nota Informativa no 13/2020 - SE/GAB/SE/MS. Manual de Recomendações para a Assistência à Gestante e Puérpera frente à Pandemia de Covid-19. Brasil, 2020. Disponível em: https://portaldeboaspraticas.iff.fiocruz.br/atencao-mulher/manual-de-recomendacoes-para-a-assistencia-agestante-e-puerpera-frente-a-pandemia-de-covid-19/. Acesso em: 22 mai. 2021.

4. MINISTÉRIO DA SAÚDE. Atenção ao pré-natal de baixo risco. Série A. Normas e Manuais Técnicos Cadernos de Atenção Básica, $\quad n^{\circ} \quad 32 \quad$ Brasil, $2012 . \quad$ Disponível em: http://bvsms.saude.gov.br/bvs/publicacoes/cadernos_atencao_basica_32_prenatal.pdf. Acessado em: 25 de maio de 2021.

5. CASAGRANDE M, et al. The enemy who sealed the world: effects quarantine due to the COVID-19 on sleep quality, anxiety, and psychological distress in the Italian population. Sleep Medicine, 2020; 75: 12-20.

6. CASTRO P, et al. Covid-19 and Pregnancy: An Overview. Revista Brasileira de Ginecologia e Obstetrícia, 2020; 42(7): 420-426.

7. CHISINI LA, et. al. Impact of the COVID-19 pandemic on Prenatal, Diabetes and medical appointments in the Brazilian National Health System. Revista Brasileira de Epidemiologia, 2021; 24: e210013.

8. CHIVERS BR, et al. Perinatal distress during COVID-19: a thematic analysis of an online parenting forum. Journal of Medical Internet Research, 2020; 22(9): e22002.

9. DING W, et al. Knowledge, attitudes, practices, and influencing factors of anxiety among pregnant women in Wuhan during the outbreak of COVID-19: a cross-sectional study. BMC Pregnancy and Childbirth; 2021; 21(80): 1-9.

10. FEDERAÇÃO BRASILEIRA DAS ASSOCIAÇÕES DE GINECOLOGIA E OBSTETRÍCIA (FEBRASGO). Protocolo de atendimento no parto, puerpério e abortamento durante a pandemia da COVID-19. Brasil, 2020. Disponível em: https://www.febrasgo.org.br/en/covid19/item/1028-protocolo-de-atendimento-no-parto-puerperio-e-abortamentodurante-a-pandemia-da-covid-19. Acesso em:08 de junho de 2021.

11. FUNDAÇÃO OSWALDO CRUZ (FIOCRUZ). Covid-19: mortes de grávidas e puérperas dobram em 2021. Brasil, 2021. Disponível em: https://dssbr.ensp.fiocruz.br/covid-19-mortes-de-gravidas-e-puerperas-dobram-em-2021-2/. Acessado em: 08 de junho de 2021.

12. FONTANA AP, et al. Pré-natal: a visão das gestantes e puérperas usuárias do serviço de saúde pública. Revista Educação em Saúde, 2017; 5(2): 72-78.

13. GONZALEZ RC, ALDERDICE F. The COVID-19 pandemic and perinatal mental health. Journal of Reproductive and Infant Psychology, 2020; 8(3):223-225.

14. HESSAMI K, et al. COVID-19 pandemic and maternal mental health: a systematic review and meta-analysis. The Journal of Maternal-Fetal \& Neonatal Medicine, 2020; 34: 1-9.

15. MALTA DC, et al. A pandemia da COVID-19 e as mudanças no estilo de vida dos brasileiros adultos: um estudo transversal. Revista de Epidemiologia e Serviços de Saúde, 2020; 29(4): e2020407.

16. NAKAMURA-PEREIRA M, et al. COVID-19 and Maternal Death in Brazil: An Invisible Tragedy. Revista Brasileira de Ginecologia e Obstetrícia, 2020; 42(8): 445-447.

17. PAIXAO JN, et al. A solidão materna diante das novas orientações em tempos de SARS-COV-2: um recorte brasileiro. Revista Gaúcha de Enfermagem, 2021; 42: 1-13.

18. PAZ MS, et al. Barreiras impostas na relação entre puérperas e recém-nascidos no cenário da pandemia do COVID19. Revista Brasileira de Saúde Materno Infantil, 2021;21(Supl. 1): 233-236.

19. QUEADAN F, et al. The risk of clinical complications and death among pregnant women with COVID-19 in the Cerner COVID-19 cohort: a retrospective analysis. BMC Pregnancy and Childbirth, 2021; 21: 1-14.

20. RAMALHO C. COVID-19 na gravidez, o que sabemos? Acta Obstetrica e Ginecologica Portuguesa, 2020; 14(1): 67.

21. RASMUSSEN SA, JAMIESON DJ. Coronavirus Disease 2019 (COVID-19) and Pregnancy: Responding to a Rapidly Evolving Situation. Obstetrics \& Gynecology, 2020; 135(5): 999-1002.

22. SILVA HN, et al. Efeitos da pandemia no novo Coronavírus na saúde mental de indivíduos e coletividades. Journal of Nursing and Health, 2020; 10: e20104007. 
23. SILVA J, MELO MQ. Um espelho de duas faces: ser ou não ser mãe? Revista Polis e Psique, 2020; 10(1): 85-106.

24. SOUZA AR, AMORIM MR. Mortalidade materna pela COVID-19 no Brasil. Revista Brasileira de Saúde Materno Infantil, 2021; 21: S257- S261.

25. SOUZA AR, et al. Women's mental health in times of COVID-1 9. Revista Brasileira de Saúde Materno Infantil, 2020; 20(3): 659-661.

26. SANTOS DS, et al. Disproportionate impact of COVID-19 among pregnant and postpartum Black Women in Brazil through structural racism lens. Clinical Infectious Diseases, 2020: 1-9.

27. TAKEMOTO MS, et al. Clinical characteristics and risk factors for mortality in obstetric patients with severe COVID19 in Brazil: a surveillance database analysis. An International Journal of Obstetrics and Gynaecology, 2020; 127(3): e161826.

28. TRAPANI JÚNIOR A, et al. Childbirth, Puerperium and Abortion Care Protocol during the COVID-19 Pandemic. Revista Brasileira de Ginecologia e Obstetrícia, 2020; 42(6): 349-355. 